\title{
O uso de óleos essenciais associado à fisioterapia para o alívio da dor na
}

\section{dismenorreia: uma revisão sistemática}

\author{
Use of essential oils associated with physiotherapy for pain relief in dysmenorrhea: a systematic \\ review
}

EI uso de aceites esenciales asociados con la fisioterapia para el alivio del dolor en la dismenorrea: una revisión sistemática

Recebido: 11/08/2021 | Revisado: 16/08/2021 | Aceito: 18/08/2021 | Publicado: 22/08/2021

Mona Indianara da Costa Aragão

ORCID: https://orcid.org/0000-0003-2557-9912 Universidade Federal do Delta do Parnaíba, Brasil

E-mail: monna.aragao@gmail.com

Sabrina Amorim Correia

ORCID: https://orcid.org/0000-0002-7527-3877 Universidade Federal do Delta do Parnaíba, Brasi E-mail: sabrinna.amorim7@gmail.com

Sylvia Christina Leite Gomes

ORCID: https://orcid.org/0000-0003-1507-3838 Universidade Federal do Delta do Parnaíba, Brasil E-mail: sylvia.clg@gmail.com

Amanda Silva Pereira

ORCID: https://orcid.org/0000-0002-8905-9468 Universidade Federal do Delta do Parnaíba, Brasil

E-mail: amandapereirasilva17@outlook.com

Kryzia de Moraes Azevedo

ORCID: https://orcid.org/0000-0002-9183-0645 Universidade Federal do Delta do Parnaíba, Brasil E-mail: kryziaazevedo@gmail.com Williams Silva Lima

ORCID: https://orcid.org/0000-0002-4756-8213 Universidade Federal do Delta do Parnaíba, Brasil E-mail: williamssilva@ufpi.edu.br

Sávia Francisca Lopes Dias

ORCID: https://orcid.org/0000-0002-4490-8331

Universidade Federal do Delta do Parnaíba, Brasil E-mail: saviadias@ufpi.edu.br

\begin{abstract}
Resumo
Esta revisão tem como objetivo reunir evidências para identificar os possíveis benefícios do uso de OEs associado à fisioterapia para o alívio da dor na dismenorreia. Para a definição da questão de pesquisa de forma estruturada foi utilizado o formato do acrônimo PICO e o processo de busca de publicações apropriadas foi totalmente documentado pelos itens de Relatórios Preferenciais para Revisões Sistemáticas e Meta-Análise (PRISMA). Os artigos selecionados estudaram a utilização de variados OEs, sejam sozinhos ou em associação. Além da massagem como principal técnica, também foi encontrada a utilização de compressas quentes associadas a OEs para o alívio da dor. De maneira geral, os manuscritos revisados encontraram resultados positivos, mostraram que os OEs apresentam grandes benefícios no tratamento da DP. O uso dos OEs associado à fisioterapia, potencializa o tratamento e ameniza o quadro álgico nas mulheres acometidas por DP. Por outro lado, nenhum dos estudos mencionou ou apresentou dados a respeito da DS.

Palavras-chave: Dismenorreia; Óleos; Fisioterapia; Terapia manual; Dor pélvica.
\end{abstract}

\begin{abstract}
This review aims to gather evidence to identify the possible benefits of using EOs associated with physical therapy for pain relief in dysmenorrhea. To define the research question in a structured way, the acronym PICO format was used and the search process for appropriate publications was fully documented by the Preferential Reports for Systematic Reviews and Meta-Analysis (PRISMA) items. The selected articles studied the use of different EOs, either alone or in association. In addition to massage as the main technique, the use of warm compresses associated with EOs for pain relief was also found. Overall, the revised manuscripts found positive results, showing that EOs have great benefits in the treatment of PD. The use of EOs associated with physiotherapy enhances the treatment and alleviates the pain in women affected by PD. On the other hand, none of the studies mentioned or presented data about SD.
\end{abstract}

Keywords: Dysmenorrhea; Oils; Physiotherapy; Manual therapy; Pelvic pain. 


\begin{abstract}
Resumen
Esta revisión tiene como objetivo reunir pruebas para identificar los posibles beneficios del uso de los entornos operativos asociados con la fisioterapia para el alivio del dolor en la dismenorrea. Para definir la pregunta de investigación de manera estructurada, se utilizó el formato de las siglas PICO y el proceso de búsqueda de las publicaciones apropiadas se documentó en su totalidad mediante los elementos de Informes Preferidos para Revisiones Sistemáticas y Metanálisis (PRISMA). Los artículos seleccionados estudiaron el uso de varios entornos operativos, ya sea solos o en asociación. Además del masaje como técnica principal, también se encontró el uso de compresas tibias asociadas con AEs para aliviar el dolor. En general, los manuscritos revisados encontraron resultados positivos, que muestran que los AEs tienen grandes beneficios en el tratamiento de la DP. El uso de AEs asociado a fisioterapia mejora el tratamiento y alivia el dolor en mujeres afectadas por DP. Por otro lado, ninguno de los estudios mencionó o presentó datos sobre la DS.
\end{abstract}

Palabras clave: Dismenorrea; Aceites; Fisioterapia; Terapia manual; Dolor pélvico.

\title{
1. Introdução
}

A dor é uma das principais causas de sofrimento humano, compromete a qualidade de vida das pessoas e interfere no seu bem-estar físico e psicossocial (Metzger, Schetta \& Walter 2002). Muitas mulheres, durante o ciclo menstrual, apresentam dores intensas em baixo ventre. Essa síndrome dolorosa é denominada dismenorreia e é popularmente conhecida como cólica menstrual (Silva, Pereira, Silva, Lima \& Lima, 2012). Vários sintomas podem estar relacionados, como cefaleia, náuseas, vômitos, sudorese, diarreia, mastalgia, dor na região lombossacra e membros inferiores, podendo levar ao surgimento de situações de nervosismo, fadiga, vertigem e até desmaio (Nunes, et. al, 2013).

Essa condição classifica-se em dismenorreia primária (DP) e dismenorreia secundária (DS). A DP ocorre na ausência de doença pélvica; inicia-se com os ciclos ovulatórios (cerca de 6 a 12 meses após a menarca) e aparece, geralmente, umas horas antes ou no início do fluxo menstrual. É mais intensa nos primeiros dois ou três dias do fluxo e pode associar-se a náuseas, vômitos e diarreia (Rodrigues et. al, 2011). Vários fatores de risco foram associados à DP, incluindo idade precoce da menarca, períodos menstruais longos, útero extravertido, tabagismo, obesidade, consumo de álcool, estresse social e psicológico (Lefebvre, et. al 2005).

Esta doença tem alta prevalência e recorrência em mulheres. Estima-se que pelo menos 60\% sofre um episódio de dor nos dias de hemorragia menstrual (Paredes, 2013). O que explica este quadro álgico é o aumento da secreção de prostaglandinas no sangue menstrual que intensifica as contrações uterinas normais, promovendo a diminuição do fluxo vascular uterino e provocando, assim, hipóxia e isquemia, responsáveis pela dor intensa (Sezeremeta, et. al, 2013).

Já a DS é decorrente de doenças pélvicas, como endometriose, fibroma, miomas e malformações uterinas. Geralmente, inicia anos após a menarca e o uso do dispositivo intrauterino (DIU) também pode ser um potencial causador da dor menstrual (Leite \& Nóbrega, 2008). As prostaglandinas estão envolvidas na DS e a dor provocada por sua liberação está relacionada a outros sintomas patológicos. Ambas possuem diagnóstico baseado na anamnese, no exame físico e em exames complementares (Leite, Leite, Machado, Portella \& Simões, 2010).

Segundo Araújo, Silva, Bastos e Ventura (2012), existem diversos tratamentos para a dismenorreia, podendo ser realizados com Anti-Inflamatórios Não Esteroides (AINEs), anticoncepcionais orais, vitaminas, agentes tocolíticos, acupuntura, entre outros. A primeira linha do tratamento farmacológico são os AINEs que atuam inibindo a síntese das prostaglandinas, diminuindo o fluxo menstrual, a contração miometrial e promovendo uma atenuação da dor (Fonseca \& Bagnoli, 2004). No entanto, a administração oral desses fármacos provoca efeitos colaterais, como hemorragias, gastrite, alterações renais e hematológicas (Diegoli \& Diegoli, 2007).

Nesse sentido, diversas terapias alternativas já foram propostas como agentes terapêuticos na redução da intensidade e duração da dor, bem como na diminuição do uso contínuo de medicamentos para a dismenorreia. Dentre elas, se destacam o uso de óleos essenciais (OEs) e a fisioterapia (Dias, Pereira, Oliveira, Santos \& Nunes, 2019). A fisioterapia oferece uma diversidade de recursos terapêuticos como exercícios, eletroterapia e terapia manual (Fae \& Hedioneia, 2010). Os OEs têm inúmeros efeitos 
sobre nosso organismo, atuam restaurando energias tanto no aspecto emocional quanto no aspecto físico, além de complementarem terapias convencionais e terapias alternativas (Sacco, Ferreira \& Silva, 2015).

A associação dessas intervenções pode sugerir uma possibilidade de potencializar o efeito analgésico, pois tanto o uso dos OEs, como algumas técnicas da fisioterapia, por exemplo, a terapia manual, promovem o alívio da dor através da ativação do sistema límbico, da melhora da circulação sanguínea e diminuição dos espasmos musculares (Da Silva, et al, 2020; Cassar, 2001).

Além disso, sabe-se que essas técnicas são eficazes para reduzir os sintomas quando realizadas regularmente e, portanto, necessitam ser mais disseminadas na população, já que a dismenorreia possui alta prevalência em mulheres jovens, interferindo principalmente nas atividades cotidianas, afetando sua qualidade de vida e bem-estar geral (Frare, Tomadon \& Silva, 2014). Neste contexto, esta revisão tem como objetivo reunir evidências científicas para identificar os possíveis benefícios do uso de óleos essenciais associados à fisioterapia para o alívio da dor na dismenorreia.

\section{Metodologia}

O presente estudo caracteriza-se metodologicamente como uma revisão sistemática, tipo de pesquisa que a partir de uma busca sistematizada de evidências científicas fornece resumos que contribuem para investigação acerca de um determinado tema. As revisões sistemáticas são amplamente aceitas como fonte confiável de conhecimento de pesquisa, enfatizadas como o topo da hierarquia de evidências e são os blocos de construção centrais para diretrizes clínicas e de políticas publicadas por organizações como a OMS (Clarke \& Chalmers, 2018).

O levantamento dos artigos utilizados foi realizado através das bases de dados: PubMed (MEDLINE), SciELO, LILACS e PEDro. Durante a pesquisa, foram avaliados estudos recentes, com prazo máximo de 10 anos de publicação. Para a busca de documentos foram incluídos os termos de pesquisa: "Dysmenorrhea", "Oil”, "Physical Therapy Modalities", "Pelvic Pain" e "Aromatherapy", sem restrição quanto aos idiomas para que fosse abrangido o maior número possível de estudos.

Para a definição da questão de pesquisa de forma estruturada foi utilizado o formato do acrônimo PICO, onde cada letra representa um componente da questão, P (população); I (intervenção); C (controle) e O (desfecho). O processo de busca de publicações apropriadas foi totalmente documentado pelos itens de Relatórios Preferenciais para Revisões Sistemáticas e MetaAnálise (PRISMA). Durante a seleção dos artigos, dois pesquisadores examinaram os títulos e resumos de todos os artigos recuperados nos bancos de dados eletrônicos, de forma cega e independente, para encontrar revisões potencialmente elegíveis, obedecendo aos critérios de inclusão delineados: artigos dos últimos 10 anos; ensaios clínicos randomizados e controlados que avaliam a eficácia do uso de OEs associado à fisioterapia em mulheres com dismenorreia. Foram excluídos estudos de caso, séries de casos, estudos observacionais e qualitativos, bem como aqueles que não conseguiram fornecer resultados satisfatórios para a pesquisa.

Os dados extraídos desta revisão sistemática foram incluídos em um quadro com informações descritivas de cada estudo, com os principais resultados alcançados em gráficos, de acordo com ano de publicação, objetivo, intervenção, valor de significância em relação ao controle e principais resultados.

\section{Resultados e Discussão}

De acordo com as diretrizes metodológicas para elaboração de revisão sistemática, a definição da pesquisa clínica deve ser estruturada utilizando o acrônimo PICO. A Figura 1 descreve a questão desta pesquisa seguindo os componentes do acrônimo PICO. 
Figura 1: Definição da questão de pesquisa de acordo com o Acrônimo PICO.

\begin{tabular}{|l|l|}
\hline P & Mulheres com dismenorreia primária e secundária \\
\hline I & Uso de óleos essenciais associados à fisioterapia \\
\hline C & Recursos (farmacológicos ou não) para alivio da dor na dismenorreia e/ou placebo \\
\hline O & Óleos essenciais associados à fisioterapia promovem o alivio da dor na dismenorreia \\
\hline
\end{tabular}

Fonte: Autores.

Na busca inicial, sem restrição de idiomas nas bases de dados, foram encontrados 358 artigos. Após nova seleção, foram excluídas as duplicatas e obtidos 196 estudos, dos quais sob outra triagem, foram excluídos mais 56 documentos que se enquadraram como resumos, estudos de caso, séries de casos, estudos observacionais e qualitativos. Após isso, um quantitativo de 140 artigos foi avaliado para elegibilidade, no qual 129 estudos foram analisados e excluídos por motivos de não possuir grupo controle, não ser randomizado ou não estar associados à fisioterapia. Por fim, 11 artigos foram avaliados e considerados relevantes para esse trabalho, e nenhum registro foi encontrado por meio de outras fontes (Figura 2).

O Quadro 1 dispõe desses 11 artigos após avaliação criteriosa de acordo com ano de publicação em ordem crescente; objetivo; número de participantes; intervenção e controle; instrumentos de avaliação; antes e depois da intervenção com o valor de significância $(\mathrm{p}<0,05)$ e os principais resultados.

Visando a obtenção de resultados fidedignos, a escolha dos artigos levou em consideração o número da amostra, esta variou de 40 a 120 mulheres. De acordo com Miot (2011), o planejamento amostral adequado de um estudo, depende do conhecimento da estatística e do problema investigado, para que se possa unir a significância dos testes ao significado clínico dos resultados.

No geral, a faixa etária das participantes ficou entre 16 a 35 anos de idade. Sabe-se que a prevalência da DP nas mulheres férteis diminui conforme a idade aumenta, isso pode ser esclarecido pela ação das prostaglandinas F2 (PGF2), quanto menor for o volume uterino, maior será sua concentração e mais intensa será a dor. O útero da adolescente tem um volume pequeno, fazendo com que a concentração das PGF2 sobre a musculatura uterina seja muito intensa. Conforme ocorre o crescimento etário, o volume uterino tende a aumentar gradualmente, reduzindo a concentração das PGF2 e diminuindo sua ação sobre essa musculatura (Dardes, Moraes \& Santos, 2011).

A dor sentida durante a dismenorreia é intensa e se localiza na região do baixo ventre e lombar. Ela ocorre durante a menstruação ou algumas horas antes, podendo persistir de 1 a 3 dias, interferindo diretamente na vida social e produtiva das mulheres (Gaubeca-Gilarranz, et. al, 2018). Ao analisar os artigos publicados, observa-se que o instrumento mais utilizado para mensuração dessa dor foi a EVA, importante para verificar a evolução do paciente de maneira confiável e analisar se o tratamento está sendo efetivo de acordo com o grau de melhora ou piora da dor (Tobo, et. al, 2010).

Outro aspecto que chamou atenção nessa revisão é que todos os estudos selecionados foram realizados no continente asiático. Paviani, Trigueiro e Gessner (2019) em uma pesquisa envolvendo o uso de OEs durante o trabalho de parto, também encontraram sua totalidade dos artigos oriundos da Ásia. Provavelmente, isto está relacionado ao fato da aromaterapia ser uma arte oriental utilizada há milhares de anos. No Egito, por exemplo, os OEs eram empregados em massagens de embelezamento, na proteção da pele e para evitar a decomposição de cadáveres, demonstrando que já tinham conhecimento das suas propriedades antissépticas (Hoare, 2010). O Brasil, por ser um país tropical e com uma extensa biodiversidade, poderia também investir mais nesse tipo de pesquisa.

$\mathrm{Na}$ atualidade, com uma base científica mais robusta, o uso da aromaterapia é realizado através de duas trajetórias principais de ação, a inalação direta, em que seus constituintes penetram através das fossas nasais e atingem o sistema límbico 
do cérebro e a outra trajetória é a aplicação tópica, que ocorre através de massagens, atingindo a corrente sanguínea, os tecidos e órgãos em geral (Montibeler, et. al, 2018). Como o objetivo desta revisão sistemática é associar os óleos essenciais à fisioterapia, 10 dos artigos escolhidos possuem a massagem terapêutica como intervenção e 1 com o uso de compressas quentes.

A massagem terapêutica é a forma mais eficaz de uso dos OEs devido ao aumento do fluxo sanguíneo na epiderme que promove o alívio da tensão muscular e proporciona efeitos fisiológicos na dor (Dos Santos, Silva \& Alfieri, 2020). A termoterapia, seja por frio ou calor, é um tratamento recorrente na fisioterapia para o alívio da dor na dismenorreia. O calor pode reduzir a tensão muscular, atuando por meio da teoria das comportas, dando a sensação de redução do quadro álgico intenso (Da Cunha, Nogueira \& Roque, 2012).

Nos artigos eleitos para discussão, foram estudados 9 tipos de OEs, sendo o de lavanda o mais mencionado, seguido respectivamente pelos de gengibre, gerânio, sálvia, rosa, hortelã - pimenta, manjerona, canela e cravo, sejam sozinhos ou em associação. Bakhtshirin, Abedi, Yusefizoj e Razmjooee (2014) utilizaram apenas o OE de lavanda em seu estudo, onde relataram que apesar da massagem com óleo de amêndoas no grupo placebo ter reduzido a dor, os resultados para o óleo de lavanda foram mais significativos na analgesia da DP. Hanfy e Kamel (2020) fizeram uma comparação entre o Treinamento de Relaxamento Progressivo e a massagem com OE de lavanda e rosa. Como resultado, não foram encontradas diferenças significativas entre os dois grupos estudados, demonstrando, assim, que a aromaterapia com lavanda e rosa possuem o mesmo efeito benéfico para DP que o treinamento.

Ou, et al (2012) objetivaram em sua pesquisa verificar o efeito analgésico de uma mistura de óleos (lavanda, sálvia e manjerona) na DP. Em seus resultados eles observaram que tanto a escala de classificação numérica quanto a de classificação verbal diminuíram significativamente após a intervenção no grupo dos óleos e no placebo (massagem com fragrância sintética de jojoba). No entanto, a duração da dor foi mais significativamente reduzida (de 2,4 para 1,8 dias) no grupo dos OEs. No estudo de Marzouk, El-Nemer e Baraka (2013) também utilizaram uma mistura de óleos (lavanda, rosa, canela e cravo diluídos em óleo de amêndoas). Além da intensidade da dor, foi avaliada a quantidade de sangue menstrual, onde ambos foram significativamente menores no grupo de OEs. Tais resultados sugerem que a aromaterapia é eficaz para aliviar tanto a dor menstrual como o sangramento excessivo, este último foi demonstrado através da quantidade de absorventes utilizados antes e depois da intervenção e pode ser atribuído ao efeito do OE de rosa, conhecido por regular o ciclo menstrual (Lee, Choi, Posadzki \& Ernst, 2012). 
Figura 2: Diagrama de Fluxo PRISMA.

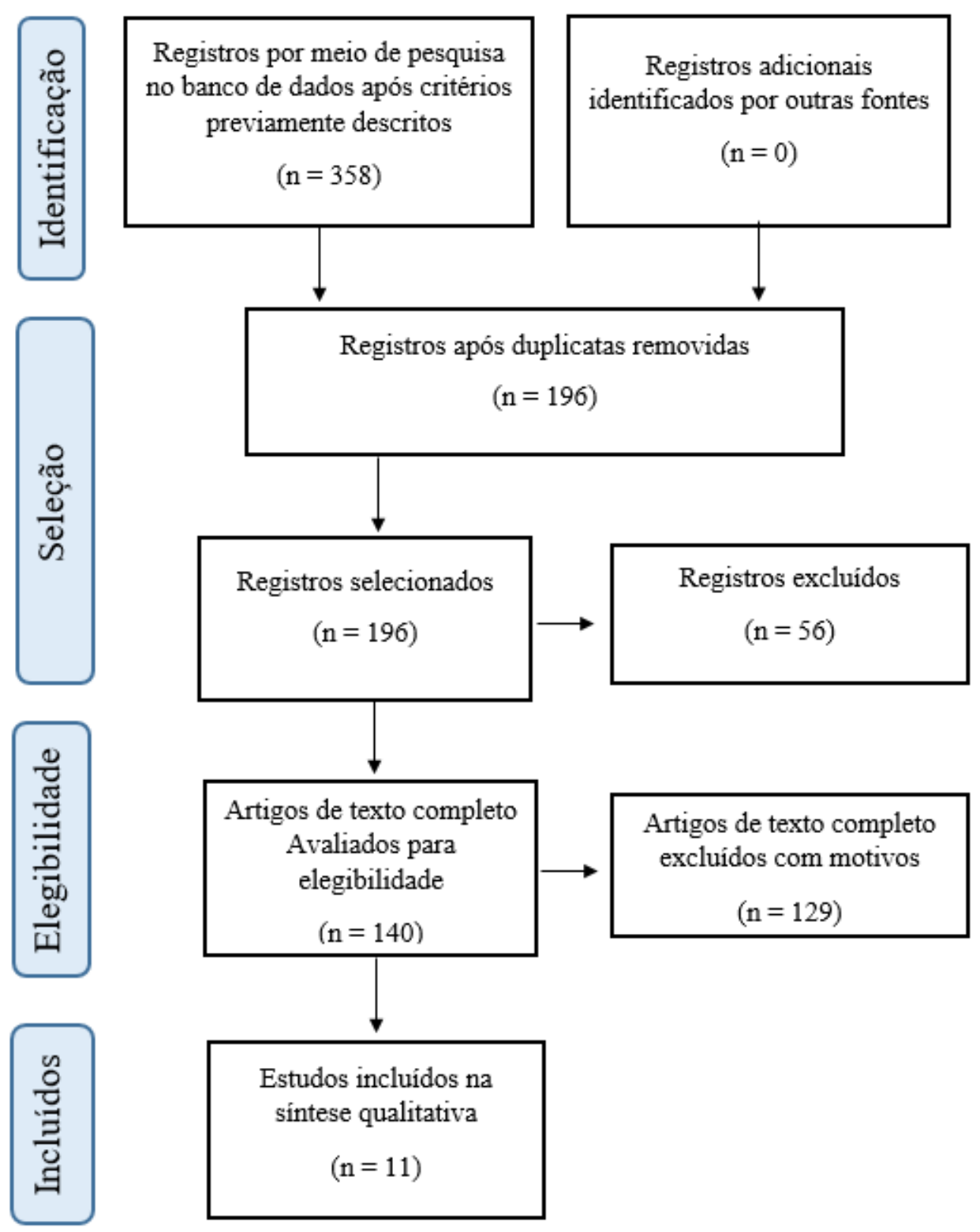

Fonte: Autores.

Pujiati, Siagian e Hardivianty (2019) escolheram o OE de lavanda para estudo, sendo em um dos grupos ele misturado a outros OEs (sálvia, gengibre e gerânio dissolvidos no óleo de amêndoas) e no outro apenas OE de lavanda (dissolvido no óleo de amêndoas). Houve uma diferença significativa entre os dois grupos, onde a mistura de OEs se mostrou mais eficaz na redução da intensidade da dor. Na literatura, vários autores defendem que o óleo de lavanda é o mais tradicional e utilizado no mundo, sendo aplicado para combater o estresse, auxiliar na insônia e promover a regeneração celular do tecido. Ele possui uma composição rica em ésteres e apresenta efeitos no alívio das cólicas menstruais e estomacais, espasmos musculares, tendinites e como sedativo (Amaral \& Silveira, 2018).

Estudos clínicos em outras condições dolorosas corroboram para o efeito analgésico do OE de lavanda. Ghods, Abforosh, Ghorbani e Asgari (2015) investigaram pacientes em hemodiálise com fístula arteriovenosa. Concluíram que a aplicação tópica do OE de lavanda diminuiu as intensidades moderadas de dor durante a inserção de agulhas de diálise. Já Costa, 
et al (2019) relataram que o uso de OEs através de massagem, entre eles o de lavanda, é capaz de relaxar pacientes com queimaduras e aliviar suas dores.

Sajjadi, Bahri e Abavisani (2018) publicaram um estudo onde se avaliou os efeitos da massagem aromática com óleo de gerânio na intensidade de dor da DP. De acordo com os resultados, a redução da dor com óleo de gerânio perfumado foi significativamente maior do que a massagem com óleo de amêndoas doce, o nível de dor foi diminuído significativamente 24 horas após a intervenção. Um ponto forte da pesquisa, foi o acompanhamento das mulheres por dois ciclos menstruais. Esse método torna os resultados mais precisos e aumenta a generalização dos resultados.

Estudos sugerem que devido a seus vários ingredientes ativos, o $\mathrm{OE}$ de gerânio tem a capacidade de estimular o sistema linfático, possuir poder antisséptico e antimicrobiano. Ele promove alto efeito positivo na redução do limiar da dor (Shokri, Khosravi, Mansouri \& Ziglari, 2011). Além disso, os efeitos antioxidantes do gerânio podem causar diminuição dos níveis de ansiedade, raiva, inquietação e depressão emocional, podendo assim, promover mais melhora a quem sofre de dismenorreia (Ali, et. al, 2015).

Em sua pesquisa, Rizk (2013) estudou três grupos, massagem apenas com OE de hortelã - pimenta, massagem apenas com OE de gengibre e o grupo controle de massagem apenas com óleo de amêndoas. Os principais resultados mostraram que, antes da intervenção, não havia diferença estatística significativa entre os grupos de estudo e controle em relação à gravidade da DP e seus sintomas associados. No entanto, os alunos que receberam massagem de aromaterapia com qualquer óleo, de hortelãpimenta ou gengibre, mostraram redução significativa na gravidade da dismenorreia e sua localização, bem como atividades de vida diária em comparação com o grupo controle, um e dois meses após a intervenção.

Outros autores também elegeram o OE de hortelã - pimenta para estudo, eles foram Kartikasari, Suryajaya \& Sintoro (2020), onde quiseram determinar se o efeito do OE de hortelã-pimenta por aromaterapia e métodos de compressas quentes pode reduzir a DM. Concluíram que a aromaterapia de hortelã-pimenta por meio de compressas quentes tem um efeito significativo na redução da EVA e do Sistema Multidimensional de Pontuação Visual (VMS), sendo um método que pode aliviar as dores menstruais.

Desde a antiguidade sabe-se que o hortelã-pimenta possui propriedades antimicrobianas, sendo muito utilizado na medicina popular. No entanto, por também possuir ativos que atuam como antiespasmódico e anti-inflamatório, costuma ser inserido no tratamento de cólicas (Sujana, Sridhar, Josthna \& Naidu, 2013). A planta Mentha piperita L. (Hortelã - pimenta) faz parte da Relação Nacional de Plantas Medicinais de Interesse ao Sistema Único de Saúde/RENISUS que tem por finalidade orientar pesquisas em fitoterapia garantindo a segurança no acesso e no uso de plantas medicinais e fitoterápicos (Benitez, Silva $\&$ Costa, 2016).

Com relação ao gengibre, ele é conhecido por suas propriedades farmacológicas e medicinais, sendo amplamente comercializado em função do seu emprego na medicina popular (Rodrigues \& Lira, 2013). Possui componentes ativos usados no OE, como o gingerol e shogol, estes impedem a ação de agentes que são altamente inflamatórios (Nicácio, et al, 2018). Estudos indicam que eles são eficazes no alívio dos sintomas da dismenorreia, uma vez que agem sobre as vias das prostaglandinas, inibindo sua síntese (Yassin, 2012). 
Research, Society and Development, v. 10, n. 11, e30101119308, 2021

(CC BY 4.0) | ISSN 2525-3409 | DOI: http://dx.doi.org/10.33448/rsd-v10i11.19308

Quadro 1: Síntese dos estudos que abordam o tema "Uso de óleos essenciais associado à fisioterapia para o alívio da dor na dismenorreia."

\begin{tabular}{|c|c|c|c|c|c|c|}
\hline Autor/ Ano & Objetivo & $\mathbf{N}$ & Intervenção/ Controle & $\begin{array}{c}\text { Instrumento } \\
\text { para } \\
\text { mensurar a } \\
\text { dor }\end{array}$ & $\begin{array}{l}\text { Antes/depois } \\
\text { valor de p }\end{array}$ & Principais resultados \\
\hline Kim et al., 2011 & 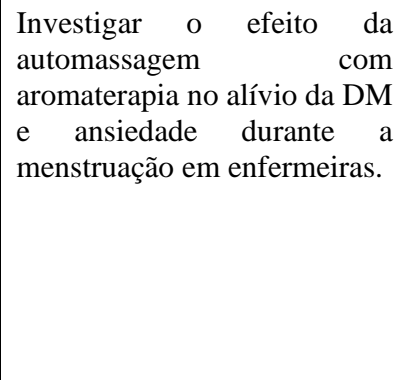 & $\begin{array}{c}\quad 55 \\
\text { enfermeiras } \\
\text { Média de idade } \\
25,0 \text { anos }\end{array}$ & $\begin{array}{l}\text { G1 (25): trataram-se com uso de OEs de } \\
\text { Rosa centifolia, Rosa damascena, } \\
\text { Salvia sclarea, Pelargonium } \\
\text { graveolens e Zingiber officinale } \\
\text { G2 (15): Massagem apenas com óleo de } \\
\text { amêndoas } \\
\text { G3 (15): não fizeram nada para aliviar } \\
\text { a dor. }\end{array}$ & EVA & $\begin{array}{c}\text { G1XG2XG3 } \\
\text { Antes da intervenção } \\
\text { p }=0,77 \\
\text { Após } 24 \text { horas } \\
\text { p< } 0,001\end{array}$ & $\begin{array}{l}\text { A automassagem no abdômen com OEs } \\
\text { aplicados topicamente pode ser um método } \\
\text { eficaz de tratamento que alivia os sintomas da } \\
\text { DM e o nível de ansiedade. }\end{array}$ \\
\hline Ou et al., 2012 & $\begin{array}{l}\text { Avaliar a eficácia da mistura } \\
\text { de OEs para pacientes com } \\
\text { DM. }\end{array}$ & $\begin{array}{l}48 \text { pacientes } \\
\text { ambulatoriais } \\
\text { Média de idade } \\
24,5 \text { anos }\end{array}$ & $\begin{array}{l}\text { G1 (24): OEs de lavanda, sálvia e } \\
\text { manjerona com uma proporção de 1: } 2 \text { : } \\
\text { 1: 1 diluída em creme de jojoba sem } \\
\text { perfume na concentração de } 3 \% \text { / } \\
\text { Massagem com a mistura de óleos. } \\
\text { G2 (24): fragrância sintética } \\
\text { adicionada ao creme de jojoba / } \\
\text { Massagem com a fragrância sintética. }\end{array}$ & $\begin{array}{l}\text { NRS 0-10 } \\
\text { VRS }\end{array}$ & $\begin{array}{c}\text { Diferença antes e } \\
\text { depois } \\
\text { NRS } 0-10 \\
\text { G1: } 1^{\circ} \text { e } 2^{\circ} \text { dia: } 2,92 \\
\text { e } 2,21(p<0,001) ; 3^{\circ} \\
\text { dia: } 1,38(p<0,05) \\
\text { G2: } 1^{\circ}, 2^{\circ} \text { e } 3^{\circ} \text { dia: } \\
\text { 1,96; } 1,62(\mathrm{p}<0,001) \\
\text { e } 0,96(\mathrm{NS}) \\
\text { VRS } \\
\text { G1: } 1^{\circ} \text { e } 2^{\circ} \text { dia: } 1,08 \text { e } \\
0,96(p<0,001) ; 3^{\circ} \\
\text { dia: } 0,63(p<0,05) \\
\text { G2 }: 1^{\circ} \text { dia: } \\
1,0(\mathrm{p}<0,001) ; 2^{\circ} \text { e } 3^{\circ} \\
\text { dia: } 0,5 \text { e } \\
0,58(\mathrm{p}<0,05)\end{array}$ & $\begin{array}{l}\text { A massagem com óleos reduziu } \\
\text { significativamente a dor e sua duração após a } \\
\text { intervenção. }\end{array}$ \\
\hline
\end{tabular}


Research, Society and Development, v. 10, n. 11, e30101119308, 2021

(CC BY 4.0) | ISSN 2525-3409 | DOI: http://dx.doi.org/10.33448/rsd-v10i11.19308

\begin{tabular}{|c|c|c|c|c|c|c|}
\hline Hur et al., 2012 & $\begin{array}{l}\text { Investigar os efeitos } \\
\text { atenuantes da MA e do } \\
\text { paracetamol na DM em } \\
\text { estudantes do ensino médio. }\end{array}$ & 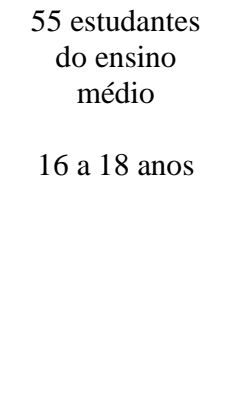 & $\begin{array}{l}\text { G1 (32) OEs de sálvia, manjerona, } \\
\text { canela, gengibre e gerânio na proporção } \\
\text { 1: 1: 0,5: 1,5: 1,5, diluído em uma base } \\
\text { de óleo de amêndoa através de } \\
\text { massagem } \\
\text { G2 (23) Paracetamol }\end{array}$ & EVA & $\begin{array}{l}\text { Mediana e intervalo } \\
\text { interquartil } \\
\text { G: redução da dor em } \\
24 \mathrm{~h} p<0,001 \\
\\
\text { G2: redução da dor } \\
\text { em } 24 \mathrm{~h} \mathrm{p}<0,001 \\
\\
\text { G1XG2 (t=-8; t=- } \\
3.69) \\
\mathrm{p}<0,001\end{array}$ & $\begin{array}{l}\text { A aromaterapia associada à massagem foi } \\
\text { considerada mais fortemente associada à } \\
\text { redução do nível de DM do que o paracetamol. }\end{array}$ \\
\hline $\begin{array}{l}\text { Marzouk, El-Nemer, } \\
\text { Baraka, 2013. }\end{array}$ & $\begin{array}{l}\text { Investigar o efeito da MA no } \\
\text { alívio da DM, na redução de } \\
\text { sua duração e sangramento } \\
\text { menstrual excessivo }\end{array}$ & $\begin{array}{l}95 \text { estudantes de } \\
\text { enfermagem } \\
17 \text { a } 20 \text { anos }\end{array}$ & $\begin{array}{l}\text { G1 (48): massagem com OEs (canela, } \\
\text { cravo, rosa, e lavanda em uma } \\
\text { proporção de 1,5: 1,5: 1: 1, } \\
\text { respectivamente, diluídos em óleo de } \\
\text { amêndoa doce com concentração final } \\
\text { de 5\%)/ massagem apenas com óleo de } \\
\text { amêndoas doce } \\
\text { G2 (47): massagem apenas com óleo de } \\
\text { amêndoas doce/ massagem com OEs } \\
\text { (canela, cravo, rosa, e lavanda em uma } \\
\text { proporção de 1,5: 1,5: 1: 1, } \\
\text { respectivamente, diluídos em óleo de } \\
\text { amêndoa doce com concentração final } \\
\text { de 5\%) }\end{array}$ & EVA & $\begin{array}{c}\text { Antes } \\
\text { G1XG2 } \\
1^{\circ} \text { dia } p=0,835 \\
2^{\circ} \text { dia } p=0,682 \\
3^{\circ} \text { dia } p=0,733 \\
1^{\text {a }} \text { fase } \\
\text { G1XG2 } \\
1^{\circ} \text { dia } p=0,013 \\
2^{\circ} \text { dia } p=0,010 \\
3^{\circ} \text { dia } p=0,006 \\
2^{a} \text { fase } \\
\text { G2XG1 } \\
1^{\circ} \text { dia } p=0,011 \\
2^{\circ} \text { dia } p=0,009 \\
3^{\circ} \text { dia } p=0,002\end{array}$ & $\begin{array}{l}\text { A aromaterapia tem um efeito significativo na } \\
\text { dor durante a menstruação e pode ser } \\
\text { considerada um tratamento seguro e eficaz no } \\
\text { tratamento para DP. }\end{array}$ \\
\hline Rizk, 2013 & $\begin{array}{l}\text { Identificar o efeito da MA } \\
\text { usando óleo de hortelã- } \\
\text { pimenta versus óleo de } \\
\text { gengibre na DP. }\end{array}$ & $\begin{array}{l}120 \text { estudantes } \\
\text { de enfermagem } \\
17 \text { a } 21 \text { anos }\end{array}$ & $\begin{array}{l}\text { G1 (40): OE de hortelã - pimenta } \\
\text { através de massagem } \\
\text { G2 (40): OE de gengibre através de } \\
\text { massagem } \\
\text { GC (40): massagem com óleo de } \\
\text { amêndoas }\end{array}$ & EVA & $\begin{array}{c}\text { Antes } \\
\text { G1XG2XGC: } \\
p=0.280 \\
\text { Após 1 mês } \\
\text { G1XG2XGC: } \\
p<0.000 \\
\\
\text { Após 2 meses } \\
\text { G1XG2XGC: } \\
p<0.000\end{array}$ & $\begin{array}{l}\text { A aromaterapia com óleos de hortelã-pimenta } \\
\text { ou gengibre é eficaz no alívio DM. }\end{array}$ \\
\hline
\end{tabular}


Research, Society and Development, v. 10, n. 11, e30101119308, 2021

(CC BY 4.0) | ISSN 2525-3409 | DOI: http://dx.doi.org/10.33448/rsd-v10i11.19308

\begin{tabular}{|c|c|c|c|c|c|c|}
\hline $\begin{array}{l}\text { Bakhtshirin et. al, } \\
2014\end{array}$ & $\begin{array}{l}\text { Investigar o efeito da MA na } \\
\text { gravidade da DP. }\end{array}$ & $\begin{array}{l}80 \text { estudantes de } \\
\text { enfermagem e } \\
\text { obstetrícia } \\
18 \text { a } 24 \text { anos }\end{array}$ & $\begin{array}{l}\text { G1 (40): OE de lavanda através de } \\
\text { massagem } \\
\text { G2 (40): placebo óleo de amêndoas doce } \\
\text { através de massagem }\end{array}$ & EVA & $\begin{array}{c}\text { Média de intensidade } \\
\text { de dor } \\
\text { G1: antes e depois } \\
\text { respectivamente } \\
(7.36 ; 3.54) p<0,001 \\
\text { G2: antes e depois } \\
\text { respectivamente } \\
(7.44 ; 6.48) \mathrm{p}<0,001 \\
\text { G1XG2: depois } \\
(3.54 ; 6.48) \\
\text { p }<0,001\end{array}$ & $\begin{array}{l}\text { Embora exista uma diferença significativa entre } \\
\text { o antes e depois da aplicação da massagem com } \\
\text { placebo, a diferença na intensidade da dor no } \\
\text { grupo com lavanda foi bem maior. Logo, a } \\
\text { massagem com óleo de lavanda pode ser usada } \\
\text { como um medicamento fitoterápico eficaz na } \\
\text { DP. }\end{array}$ \\
\hline Shahr et al., 2015 & $\begin{array}{l}\text { Avaliar o efeito da } \\
\text { automassagem } \\
\text { aromaterapêutica abdominal } \\
\text { na DP. }\end{array}$ & $\begin{array}{l}75 \text { estudantes } \\
\text { universitárias } \\
18 \text { a } 35 \text { anos }\end{array}$ & $\begin{array}{l}\text { G1 (25): aplicou a massagem com OE } \\
\text { de Rosa damascena diluída em óleo de } \\
\text { amêndoas } \\
\text { G2 (25): realizou a massagem com óleo } \\
\text { de amêndoas sem perfume } \\
\text { G3 (25): realizou apenas a massagem }\end{array}$ & EVA & $\begin{array}{c}\text { Ciclo 1: } \\
\text { Antes da intervenção } \\
\text { G1XG2XG3 } \\
\text { p = 0,114 } \\
\text { Após a intervenção } \\
\text { G1XG2XG3 } \\
\text { p = 0,581 } \\
\text { Ciclo 2: } \\
\text { Antes da intervenção } \\
\text { G1XG2XG3 } \\
\text { p = 0,064 } \\
\text { Após a intervenção } \\
\text { G1XG2XG3 } \\
\text { p }=0,000\end{array}$ & $\begin{array}{l}\text { Os resultados revelam que no } 1^{\circ} \text { ciclo, o nível } \\
\text { médio de dor diminuiu nos } 3 \text { grupos, mas foi } \\
\text { NS. } \\
\text { No segundo ciclo, também foi NS na redução da } \\
\text { dor entre os grupos } 2 \text { e } 3 \text {. Esses dados sugerem } \\
\text { que a massagem terapêutica com óleo de Rosa } \\
\text { Damascena fornece uma mudança significativa } \\
\text { na redução da dor entre os } 3 \text { grupos, com } \\
\text { probabilidade de } 99 \% \text {. }\end{array}$ \\
\hline $\begin{array}{l}\text { Sajjadi, } \quad \text { Bahri, } \\
\text { Abavisani, } 2018\end{array}$ & $\begin{array}{l}\text { Estudar os efeitos da MA com } \\
\text { óleo de gerânio na intensidade } \\
\text { da dor na DP. }\end{array}$ & $\begin{array}{l}90 \text { estudantes } \\
\text { universitárias } \\
18 \text { a } 35 \text { anos }\end{array}$ & $\begin{array}{l}\text { G1 (30): OE de gerânio através de } \\
\text { massagem } \\
\text { G2 (30): óleo de amêndoas doce } \\
\text { através de massagem } \\
\text { GC (30): sem intervenção }\end{array}$ & EVA & $\begin{array}{c}\text { Antes } \\
\text { G1XG2XGC } \\
\mathrm{p}=0.230 \\
\text { Imediatamente } \\
\text { após } \\
\text { G1XG2XGC } \\
\text { p }<0.001 \\
\text { 24h após } \\
\text { G1XG2XGC } \\
\text { p }<0.001\end{array}$ & A MA com OE de gerânio reduz a dor da DP. \\
\hline
\end{tabular}


Research, Society and Development, v. 10, n. 11, e30101119308, 2021

(CC BY 4.0) | ISSN 2525-3409 | DOI: http://dx.doi.org/10.33448/rsd-v10i11.19308

\begin{tabular}{|c|c|c|c|c|c|c|}
\hline $\begin{array}{l}\text { Pujiati,Siagian, } \\
\text { Hardivianty, } 2019 .\end{array}$ & $\begin{array}{l}\text { Realizar investigação } \\
\text { utilizando OEs de Lavanda, } \\
\text { Salva esclareia, Gengibre e } \\
\text { Gerânio através de MA para a } \\
\text { DM. }\end{array}$ & $\begin{array}{c}84 \text { estudantes de } \\
\text { enfermagem } \\
18 \text { a } 25 \text { anos }\end{array}$ & $\begin{array}{l}\text { G1 (42): OEs lavanda, Salva esclareia, } \\
\text { gengibre, gerânio com uma proporção de } \\
\text { 1: 1: 1: } 1 \text { com óleo de amêndoas através } \\
\text { de Massagem Effleurage } \\
\text { G2 (42): OE Lavanda dissolvido com } \\
\text { Óleo de Amêndoas através de Massagem } \\
\text { Effleurage }\end{array}$ & NRS 0-10 & $\begin{array}{c}\text { Mediana } \\
\text { G1:Antes: } 3 \\
\text { Depois: } 2 \\
\text { p = 0,001 } \\
\text { G2: Antes: } 3 \\
\text { Depois: } 3 \\
p=0,046 \\
\text { G1XG2: } p=0,001\end{array}$ & $\begin{array}{l}\text { OEs de lavanda, Salva esclareia, gengibre e } \\
\text { gerânio como aromaterapia através da } \\
\text { massagem foram mais eficazes na redução da } \\
\text { intensidade da DM. }\end{array}$ \\
\hline Hanfy et al., 2020 & $\begin{array}{l}\text { Estudar o } \begin{array}{l}\text { efeito do } \\
\text { treinamento de } \\
\text { relaxamento } \\
\text { progressivo }\end{array} \quad \text { versus } \\
\text { aromaterapia na DP. }\end{array}$ & $\begin{array}{l}40 \text { pacientes } \\
\text { ambulatoriais } \\
16 \text { a } 25 \text { anos }\end{array}$ & $\begin{array}{l}\text { G1 (20): Treinamento de relaxamento } \\
\text { progressivo } \\
\text { G2 (20): OE de lavanda e rosa a } 2 \% \\
\text { através de massagem }\end{array}$ & EVA e NCS & $\begin{array}{c}\text { Mediana } \\
\text { EVA } \\
\text { G1 Pré/Pós } \\
p=0,001 \\
\text { G2 Pré/Pós } \\
p=0,001 \\
\text { NCS } \\
\text { G1 Pré/Pós } \\
p=0,001 \\
\text { G2 Pré/Pós } \\
p=0,001 \\
\text { EVA G1XG2 } \\
\text { Pré: } p=0,785 \\
\text { Pós: } p=0,958 \\
\text { NCS } \\
\text { G1XG2 } \\
\text { Pré: } p=0,829 \\
\text { Pós: } p=0,149\end{array}$ & $\begin{array}{l}\text { A aromaterapia tem o mesmo efeito benéfico e } \\
\text { significativo do treinamento de relaxamentos } \\
\text { progressivos na melhora da dor na DP. }\end{array}$ \\
\hline
\end{tabular}


Research, Society and Development, v. 10, n. 11, e30101119308, 2021

(CC BY 4.0) | ISSN 2525-3409 | DOI: http://dx.doi.org/10.33448/rsd-v10i11.19308

\begin{tabular}{|c|c|c|c|c|c|c|}
\hline $\begin{array}{l}\text { Kartikasari, } \\
\text { Suryajaya,Sintoro, } \\
2020\end{array}$ & $\begin{array}{l}\text { Determinar se o efeito do OE } \\
\text { de hortelã-pimenta por MA e } \\
\text { métodos de compressas } \\
\text { quentes } \\
\text { podem reduzir a DM em } \\
\text { alunos da Faculdade de } \\
\text { Medicina da Universidade de } \\
\text { Hang Tuah Surabaya. }\end{array}$ & $\begin{array}{l}42 \text { estudantes } \\
\text { universitárias } \\
\text { Idade média } 21,0 \\
\text { anos }\end{array}$ & $\begin{array}{l}\text { G1 (21): recebeu OE de hortelã- } \\
\text { pimenta por compressas quentes } \\
\text { G2 (21): Sem intervenção }\end{array}$ & $\begin{array}{l}\text { EVA } \\
\text { VMS }\end{array}$ & $\begin{array}{c}\text { G1XG2 } \\
\text { EVA } \\
\mathrm{p}=0,001 \\
\text { VMS } \\
\mathrm{p}=0,001\end{array}$ & $\begin{array}{l}\text { O uso da aromaterapia com hortelã-pimenta } \\
\text { por compressas quentes afetou a redução EVA } \\
\text { e VMS. }\end{array}$ \\
\hline
\end{tabular}

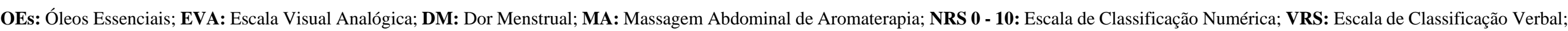
NS: Não Significativo; DP: Dismenorreia Primária; NSC: Nível de Cortisol Sérico; VMS: Sistema Multidimensional de Pontuação Visual.

Fonte: Autores 
A eficácia do alívio da dor na dismenorreia pelo gengibre foi comprovada por vários autores deste estudo, onde todos o utilizaram em associação com outros óleos. Além de Kartikasari, Suryajaya e Sintoro (2020) que o associou com hortelã pimenta e Pujiati, Siagian e Hardivianty (2019) a gerânio, sálvia e lavanda. Ainda pode-se citar Kim, Lee, Yang e Hur (2011) com Rosa centifolia, Rosa damascena, sálvia, gerânio e Hur, Lee, Seong e Lee (2012) associando com sálvia, manjerona, canela e gerânio. Torna-se importante ressaltar, que neste último o uso da combinação de OEs foi comparado com o Paracetamol, medicamento de primeira escolha no alívio de dores leves a moderadas, como cefaleias, dores musculares, estados gripais, dores leves de artrite, dor de dente, cólicas menstruais e redução da febre, sendo eficaz em todas as idades e que há anos vem provando sua eficácia clínica e segurança como analgésico (Klotz, 2012), mesmo assim, como resultado, a combinação de OEs se mostrou mais eficiente.

Dois autores tiveram como método de intervenção a automassagem com a utilização da OE de Rosa damascena. Kim, Lee, Yang e Hur. (2011), já mencionados anteriormente, utilizaram uma associação de óleos e Sharh, Saadat, Kheirkhah e Saadat (2015) que utilizaram o OE sem associação e realizaram uma intervenção que envolvia três grupos: massagem com OE de rosa, massagem com óleo de amêndoa sem perfume e apenas massagem. Como resultado, os dados sugeriram que a massagem terapêutica com OE de rosa fornece uma mudança significativa na redução da dor entre os 3 grupos, com probabilidade de $99 \%$. É de suma importância esclarecer que em ambos os estudos todas as participantes foram previamente treinadas para aplicar automassagem em casa.

Por muito tempo, a principal aplicação do óleo de rosa foi na indústria de cosméticos, mas atualmente existe um interesse crescente devido ao seu potencial farmacológico. Hoje, além da aromaterapia, ele já está fazendo parte de algumas formulações de anti-inflamatório, reguladores respiratórios e tratamento para esclerose (Loulanski \& Loulanski, 2014).

Outros OEs mencionados nos estudos selecionados, foram: canela, muito utilizado como diurético, analgésico, poderoso antisséptico, e antiespasmódico (Andrei \& Del Comune, 2005); cravo, um antioxidante natural, analgésico, anti-inflamatório, cicatrizante, e que ainda possui diversos benefícios sobre o sistema mente-corpo (Daniel et al, 2009); sálvia que apresenta propriedades antissépticas e anti-inflamatórias (Baudoux, 2018), sendo muito utilizada no tratamento da tensão pré-menstrual e sintomas do climatério (Pereira, et al, 2017); manjerona utilizado para combater a tosse, problemas na bexiga e cólicas gastrointestinais. Além de se apresentar eficiente como antioxidante, tanto que essa propriedade foi comparada à conhecida atividade da vitamina $\mathrm{E}$ (Jun, et al, 2001).

As práticas integrativas e complementares, como a aromaterapia, são utilizadas desde a antiguidade pelos mais diversos povos no tratamento da saúde, porém, são poucos os estudos que compreendem de forma aprofundada seus mecanismos biológicos (Price \& Price, 2012). No Brasil, as terapias não farmacológicas foram estabelecidas no Sistema Único de Saúde (SUS), através da Política Nacional de Práticas Integrativas e Complementares (PNPIC), pela Portaria GM/MS nº 9712, da qual a aromaterapia faz parte e consiste na aplicação de OEs com finalidade terapêutica (Brasil, 2018).

Como os OEs são altamente concentrados, eles devem ser usados com óleos carreadores, como amêndoa, óleo de jojoba ou azeite, caso contrário, eles terão efeitos colaterais, como irritação na pele (Sinha, 2010). Os artigos citados nesta revisão revelaram que o uso de aromaterapia, seja por massagem aromaterapêutica ou por compressas quentes, são eficazes para o alívio das dores menstruais e alguns outros sintomas da dismenorreia.

\section{Considerações Finais}

De maneira geral, a revisão sistemática realizada no presente manuscrito, mostra que os OEs apresentam grandes benefícios e potencial para aplicação no tratamento das dores menstruais em mulheres que sofrem de DP. Ressalta-se, ainda, que nenhum dos estudos selecionados mencionou ou apresentou dados a respeito da DS. 
As evidências encontradas sugerem que o uso dos OEs, sejam isolados ou em associação, são viáveis na Fisioterapia como forma de potencializar o tratamento, amenizar o quadro álgico e melhorar a qualidade de vida das mulheres acometidas por DP.

Além de ser indicado dentro das Práticas Integrativas e Complementares, o uso de OEs é um método de fácil utilização, baixo custo e, se bem administrado, sem efeitos colaterais. No entanto, devido à escassez de trabalhos sobre esse tema, no que diz respeito às propriedades e aplicação, são necessários mais estudos para aprimorar o conhecimento de acadêmicos e profissionais da área de saúde e, assim, passem a ser utilizados na prática clínica.

Para estudos futuros, sugere-se a realização de ensaios clínicos controlados e randomizados onde sejam investigadas e descritas minuciosamente a dosagem de diluição para aplicação, as reaçães adversas e o período do ciclo menstrual onde o uso dos OEs tenha maior efetividade na redução da dor, além de pesquisas que visem um maior conhecimento sobre seus mecanismos biológicos. Através desses dados, a utilização da aromaterapia terá um maior respaldo científico e poderá ser reproduzida de forma mais técnica e efetiva no tratamento da dismenorreia.

\section{Referências}

Ali, B., Al-Wabel, N. A., Shams, S., Ahamad, A., Khan, S. A., \& Anwar, F. (2015). Essential oils used in aromatherapy: A systemic review. Asian Pacific Journal of Tropical Biomedicine, 5 (8), 601-611.

Amaral, F., \& Silveira, C. (2018). Manual técnico: cuidados naturais da cabeça aos pés. Wnf.

Andrei, P., \& Del Comune, A. P. (2005). Aromaterapia e suas aplicações. Centro Universitário São Camilo, 11 (4), $57-68$.

Araújo, L. M. D., Silva, J. M. N. D., Bastos, W. T., \& Ventura, P. L. (2012). Diminuição da dor em mulheres com dismenorreia primária, tratadas pelo método Pilates. Revista Dor, 13, 119-123.

Bakhtshirin, F., Abedi, S., YusefiZoj, P., \& Razmjooee, D. (2015). The effect of aromatherapy massage with lavender oil on severity of primary dysmenorrhea in Arsanjan students. Iranian journal of nursing and midwifery research, 20 (1), 156.

Baudoux, D. (2018). O Grande Manual de Aromaterapia de Dominique Baudoux. Trad.: Mayra Corrêa e Castro. Belo Horizonte. Editora Lazslo.

Benitez, L. B., da Silva, C. D. M., \& da Costa Alvares, L. (2016). Utilização da hortelã-pimenta como agente no controle de infecções relacionadas à assistência à saúde (IRAS). Scientia Plena, 12 (12).

Brasil. Ministério da Saúde. Departamento de Ciência e Tecnologia (2012). Diretrizes metodológicas: elaboração de revisão sistemática e metanálise de ensaios clínicos randomizados [Internet]. Brasília(DF): Editora do Ministério da Saúde.

Brasil. Ministério da Saúde (BR). (2018). Portaria 702, de 21 de março de 2018. Altera a Portaria de Consolidação n 2/GM/MS, de 28 de setembro de 2017, para incluir novas práticas na Política Nacional de Práticas Integrativas e Complementares-PNPIC.

Cassar, M. P. (2001). Manual de massagem terapêutica. Barueri: Editora Manole.

Clarke, M., \& Chalmers, I. (2018). Reflections on the history of systematic reviews. BMJ Evidence Based Medicine, n. 23, $121-122$.

Costa, I. B., Dantas, D. V., Dantas, R. A. N., Oliveira, S. P., Aiquoc, K. M., \& Sarmento, S. D. G. (2019). Terapias não farmacológicas para o manejo da dor em vítima de queimaduras: uma revisão sistemática. Revista Inspirar: movimento e saúde, 19 (2), 1-20.

Da Cunha, A. P., Nogueira, M. T., \& Roque, O. R. (2012). Plantas aromáticas e óleos essenciais: composição e aplicações. Fundação Calouste Gulbenkian.

Da Silva, I. T. S., de Araújo, A. C., de Medeiros, Y. E., da Costa Santos, R. S., Góis, M. M. D. C. D., \& da Silva, R. A. R. (2020). O uso da aromaterapia no contexto da enfermagem: uma revisão integrativa. Revista Eletrônica de Enfermagem, 22.

Daniel, A. N., Sartoretto, S. M., Schmidt, G., Caparroz-Assef, S. M., Bersani-Amado, C. A., \& Cuman, R. K. N. (2009). Anti-inflammatory and antinociceptive activities A of eugenol essential oil in experimental animal models. Revista Brasileira de Farmacognosia, 19, $212-217$.

Dardes, R. D. C. D. M., Moraes, A. S., \& Santos, M. B. (2011). Dismenorreia. RBM rev. bras. med.

Dias, S. F. L., Pereira, L. C. A., Oliveira, A. P. D., Santos, R. F. D., \& Nunes, L. C. C. (2019). Scientific and technological prospection on transdermal formulations and complementary therapies for the treatment of primary dysmenorrhea. Expert opinion on therapeutic patents, 29 (2), $115-126$.

Diegoli, C. A., \& Diegoli, M. S. C. (2007). Saúde da Mulher Dismenorréia. Revista Brasileira de Medicina-RBM, 64 (3).

Dos Santos, G. K. A., Silva, N. C. D. O. V., \& Alfieri, F. M. (2020). Efeitos da compressa fria versus quente sobre a dor em universitárias com dismenorreia primária. BrJP, 3, 25-28. 
Fae, A., \& Pivetta, H. M. F. (2010). Efeitos da massagem do tecido conjuntivo e cinesioterapia no tratamento da dismenorréia primária. Fisioterapia Brasil, 11 (2).

Fonseca, A. M., \& Bagnoli, V. R. (2004). Como diagnosticar e tratar: dismenorréia. Revista Brasileira de Medicina, 62, 113-6.

Frare, J. C., Tomadon, A., \& Silva, J. R. (2014). Prevalência da dismenorreia e seu efeito na qualidade de vida entre mulheres jovens. Revista Brasileira de Ciências da Saúde, 12 (39), 15-20.

Gaubeca-Gilarranz, A., Fernández-De-Las-Peñas, C., Medina-Torres, J. R., Seoane-Ruiz, J. M., Company-Palonés, A., Cleland, J. A., \& Arias-Buría, J. L. (2018). Effectiveness of dry needling of rectus abdominis trigger points for the treatment of primary dysmenorrhoea: a randomised parallel-group trial. Acupuncture in Medicine, 36 (5), 302-310.

Ghods, A. A., Abforosh, N. H., Ghorbani, R., \& Asgari, M. R. (2015). The effect of topical application of lavender essential oil on the intensity of pain caused by the insertion of dialysis needles in hemodialysis patients: a randomized clinical trial. Complementary therapies in medicine, 23 (3), 325-330.

Hanfy, H. M., Kamel, H. E. H., Kamal, W. M., \& Mahmoud, S. M. (2020). The effect of progressive relaxation training versus aromatherapy on primary dysmenorrhea. Egypt, Med. J. Cairo Univ, 88 (2), 577-582.

Hoare, J., \& Wilson, S. (2010). Guia completo de aromaterapia: um curso estruturado para alcançar a excelência profissional. Pensamento.

Hur, M. H., Lee, M. S., Seong, K. Y., \& Lee, M. K. (2012). Aromatherapy massage on the abdomen for alleviating menstrual pain in high school girls: a preliminary controlled clinical study. Evidence-Based Complementary and Alternative Medicine, 2012.

Jun, W. J., Han, B. K., Yu, K. W., Kim, M. S., Chang, I. S., Kim, H. Y., \& Cho, H. Y. (2001). Antioxidant effects of Origanum majorana L. on superoxide anion radicals. Food Chemistry, 75 (4), 439-444.

Kartikasari, R. (2020). Effect Of Peppermint Aromatherapy On Menstruation Scale Of Pain In Fakultas Kedokteran Hang Tuah Surabaya Students. Jurnal AgriTek: Jurnal Penelitian Ilmu-Ilmu Eksakta, 21 (1), 10-13.

Kim, Y. J., Lee, M. S., Yang, Y. S., \& Hur, M. H. (2011). Self-aromatherapy massage of the abdomen for the reduction of menstrual pain and anxiety during menstruation in nurses: a placebo-controlled clinical trial. European journal of integrative medicine, 3 (3), e165-e168.

Klotz, U. (2012). Paracetamol (acetaminophen)-a popular and widely used nonopioid analgesic. Arzneimittelforschung, 62 (08), 355-359.

Lee, M. S., Choi, J., Posadzki, P., \& Ernst, E. (2012). Aromatherapy for health care: an overview of systematic reviews. Maturitas, 71 (3), $257-260$.

Lefebvre, G., Pinsonneault, O., Antao, V., Black, A., Burnett, M., Feldman, K., ... \& Robert, M. (2005). Primary dysmenorrhea consensus guideline. J Obstet Gynaecol Can, 27 (12), 1117-46.

Leite, M. C. A., Leite, C. A., Machado, R. F. F., Portella, A. S. J. A., \& Simões, B. N. (2010). Dismenorréia: uma visão atual. Anais do, 11.

Leite, M. C. A., \& da Nóbrega, M. M. (2008). A dismenorréia primária como problema de saúde pública. Revista de Ciências da Saúde Nova Esperança, 6 (1), $8-19$.

Loulanski, V., \& Loulanski, T. (2014). The heritization of Bulgarian rose. Acta Geographica Slovenica, 54 (2), 401-410.

Marzouk, T. M., El-Nemer, A. M., \& Baraka, H. N. (2013). The effect of aromatherapy abdominal massage on alleviating menstrual pain in nursing students: a prospective randomized cross-over study. Evidence-Based Complementary and Alternative Medicine, 2013.

Metzger, C., Schwetta, M., Walter, C., Müller, A., \& Martins, M. M. S. (2002). Cuidados de Enfermagem e dor.

Miot, H. A. (2011). Tamanho da amostra em estudos clínicos e experimentais. Jornal Vascular Brasileiro, 10, 275-278.

Montibeler, J., Domingos, T. D. S., Braga, E. M., Gnatta, J. R., Kurebayashi, L. F. S., \& Kurebayashi, A. K. (2018). Efetividade da massagem com aromaterapia no estresse da equipe de enfermagem do centro cirúrgico: estudo-piloto. Revista da Escola de Enfermagem da USP, 52.

Nicácio, G. L., Moura, S. C., Costa, J. V. D. J., Sena, C. R., Cruz, T. B. F., Lopes, G. N. M., \& Cecílio, A. B. (2018). Breve revisão sobre as propriedades fitoterápicas do Zingiber officinale Roscoe - o gengibre. Sinapse múltipla, 7 (2), 74-80.

Oliveira Nunes, J. M., do Amaral Rodrigues, J., de Freitas Moura, M. S., Batista, S. R. C., Coutinho, S. K. S. F., Hazime, F. A., \& dos Reis Barbosa, A. L. (2013). Prevalência de dismenorreia em universitárias e sua relação com absenteísmo escolar, exercício físico e uso de medicamentos. Revista Brasileira em Promoção da Saúde, 26 (3), 381-386.

Ou, M. C., Hsu, T. F., Lai, A. C., Lin, Y. T., \& Lin, C. C. (2012). Pain relief assessment by aromatic essential oil massage on outpatients with primary dysmenorrhea: A randomized, double-blind clinical trial. Journal of Obstetrics and Gynaecology Research, 38 (5), 817-822.

Paredes, J. S., Jaramillo, A. M., \& Paz, C. S. P. S. (2013). Factores que condicionan la dismenorrea en adolescentes, según estrato socioeconómico. Revista Peruana de Ginecología y obstetricia, 59(2), 95-100.

Paviani, B. A., Trigueiro, T. H., \& Gessner, R. (2019). O uso de óleos essenciais no trabalho de parto e parto: revisão de escopo. Revista Mineira de Enfermagem, 23, 1-8.

Pereira, A., Bertoni, B., Silva, C., Ferro, D., Carmona, F., Dandaro, I., \& Doneida, V. (2017). Formulário de preparação extemporânea: farmácia da naturezachás medicinais.

Price, S., \& Price, L. (2011). Aromatherapy for health professionals E-book. Elsevier Health Sciences. 
Research, Society and Development, v. 10, n. 11, e30101119308, 2021

(CC BY 4.0) | ISSN 2525-3409 | DOI: http://dx.doi.org/10.33448/rsd-v10i11.19308

Pujiati, W., Siagian, Y., \& Hardivianty, C. (2018). Application of Essential Oils: Lavender, Clary Sage, Ginger and Geranium as Aromatherapy through Effleurage Massage for Menstrual Pain. International Journal of Science and Research (IJSR).

Rizk, S. A. (2013). Effect of aromatherapy abdominal massage using peppermint versus ginger oils on primary dysmenorrhea among adolescent girls. Journal of American Science, 9 (11), 497-505.

Rodrigues, A. C., Gala, S., Neves, A., Pinto, C., Meirelles, C., Frutuoso, C., \& Vítor, M. E. (2011). Dismenorreia em adolescentes e jovens adultas: prevalência, factores associados e limitações na vida diária. Acta Med Port, 24 (S2), 383-92.

Rodrigues, M. L., \& Lira, R. K. (2013). Perfil fitoquímico e biológico do extrato hidroalcoólico dos rizomas do gengibre (Zingiber officinale Roscoe). SaBiosRevista de Saúde e Biologia, 8 (1).

Sacco, P. R., Ferreira, G. C. G. B., \& Silva, A. C. C. D. (2015). Aromaterapia no auxílio do combate ao estresse: bem-estar e qualidade de vida. Revista científica da FHO| UNIARARAS, 3 (1).

Sajjadi, M., Bahri, N., \& Abavisani, M. (2018). Aromatherapy massage with geranium essence for pain reduction of primary dysmenorrhea: a double blind clinical trial. The Iranian Journal of Obstetrics, Gynecology and Infertility, 20 (12), 50-57.

Sezeremeta, D. C., dos Santos Carvalho, M. S., Vrecchi, M. R., Marafon, R. G. C., Crespilho, L. C., Pagotto, J. P., \& Mortean, E. D. C. M. (2013). Dismenorreia: Ocorrência na vida de acadêmicas da área de saúde. Journal of Health Sciences, 15 (2).

Shahr, H., Saadat, M., Kheirkhah, M., \& Saadat, E. (2015). The effect of self-aromatherapy massage of the abdomen on the primary dysmenorrhoea. Journal of Obstetrics and Gynaecology, 35 (4), 382-385.

Shokri, H., Khosravi, A. R., Mansouri, M., \& Ziglari, T. (2011). Effects of Zataria multiflora and Geranium pelargonium essential oils on growth-inhibiting of some toxigenic fungi. Iranian Journal of Veterinary Research, 12(3), 247-251.

Silva, A. B., Pereira, A. O., Silva, S. P., Lima, C. R. J., \& Lima, A. B. (2012). Correlação entre as alterações posturais e a dismenorreia primária em mulheres jovens na faixa etária de 18 a 25 anos. Revista Científica do Unisalesiano-Lins-SP, 3 (6), 253-265.

Sinha, A. G. (2010). Principle and practice of therapeutic massage. Editor: Textbook of massage. India: Jaypee Brothers Medical Publishers, p.8-23.

Sujana, P., Sridhar, T. M., Josthna, P., \& Naidu, C. V. (2013). Antibacterial activity and phytochemical analysis of Mentha piperita L.(Peppermint) - An importante multipurpose medicinal plant. Am J Plant Sci; 4 (1):77-83.

Tobo, A., Khouri, M., Cordeiro, Q., Lima, M. D. C., Brito Junior, C. A., \& Battistella, L. R. (2010). Estudo do tratamento da lombalgia crônica por meio da Escola de Postura. Acta fisiatrica, 17 (3), 112-6.

Yassin, S. A. (2012). Herbal remedy used by rural adolescent girls with menstrual disorders. J Am Sci, 8 (1), 467-73. 